\title{
Análise da compliância e gradiente timpanométrico em lactentes com refluxo
}

\author{
Analysis of compliance and tympanometric gradient in infants \\ with reflux
}

\author{
Elizângela Dias Camboim ${ }^{1}$, Renata Coelho Scharlach ${ }^{2}$, Maiara Cristine Oliveira de Almeida ${ }^{1}$, \\ Dênis Vasconcelos ${ }^{3}$, Marisa Frasson de Azevedo ${ }^{4}$
}

\begin{abstract}
RESUMO
Objetivo: Analisar e comparar o gradiente timpanométrico e a compliância obtida nas sondas de $226 \mathrm{~Hz}$ e $1 \mathrm{kHz}$ entre os grupos de lactentes com e sem refluxo gastroesofágico. Métodos: Cento e dezoito lactentes a termo e pré-termo, de recém-nascidos a 6 meses de idade - 63 com diagnóstico clínico de refluxo gastroesofágico fisiológico realizado por pediatras ou gastropediatras e 55 sem refluxo -, foram submetidos a timpanometria com sondas de $226 \mathrm{~Hz}$ e $1 \mathrm{kHz}$. Resultados: Foram observados maiores valores de compliância com sonda de $1 \mathrm{kHz}$ em ambos os grupos. Ao se comparar a média de compliância entre os grupos, observou-se que o grupo sem refluxo apresentou maiores valores. A média dos valores do gradiente timpanométrico foi maior no grupo sem refluxo, quando comparada ao grupo com refluxo. Conclusão: A sonda de $1 \mathrm{kHz}$ apresenta maior compliância em lactentes com e sem refluxo, em relação à sonda de $226 \mathrm{~Hz}$. Lactentes com refluxo apresentam compliância dentro dos padrões de normalidade, porém apresentando menor compliância quando comparados com lactentes sem refluxo. Com relação ao gradiente, lactentes com refluxo apresentam valores alterados e/ou dentro dos padrões limítrofes da normalidade, em ambas as orelhas.
\end{abstract}

Descritores: Testes de impedância acústica; Orelha média; Refluxo gastroesofágico; Lactente; Audição

\section{INTRODUÇÃO}

O refluxo gastroesofágico (RGE) é um processo fisiológico comum, principalmente em lactentes ${ }^{(1)}$. Ocorre em aproximadamente $50 \%$ das crianças abaixo de 3 meses, em $67 \%$ na faixa etária de 4 a 6 meses e em 5\% entre 10 e 12 meses de vida. Pode ser classificado em fisiológico e patológico. O fisiológico provoca apenas regurgitação e/ou vômitos, não interferindo no desenvolvimento normal da criança. Já o patológico, além dos vômitos e/ou regurgitações, outras manifestações clínicas estão presentes, como parada do crescimento, sinais sugestivos de inflamação do esôfago (esofagite), principalmente irritabilida-

Trabalho realizado na Universidade Estadual de Ciências da Saúde de Alagoas UNCISAL - Maceió (AL), Brasil.

Conflito de interesses: Não

(1) Curso de Fonoaudiologia, Universidade Estadual de Ciências da Saúde de Alagoas - UNCISAL - Maceió (AL), Brasil.

(2) Disciplina de Distúrbios da Audição, Departamento de Fonoaudiologia, Universidade Federal de São Paulo - UNIFESP - São Paulo (SP), Brasil.

(3) Instituto da Mulher, Hospital Santa Juliana de Alagoas.

(4) Departamento de Fonoaudiologia, Universidade Federal de São Paulo UNIFESP - São Paulo (SP), Brasil.

Endereço para correspondência: Elizângela Dias Camboim. R. Coronel Alcides de Barros Ferreira, 26/301, Jatiúca, Maceió (AL), Brasil, CEP:

57036-480. E-mail: elicamboim@yahoo.com.br

Recebido em: 14/7/2011; Aceito em: 10/11/2011 de, choro persistente, e dificuldade para dormir, encontradas principalmente em lactentes pequenos $\mathrm{s}^{(2-4)}$. O material refluído contém ácido clorídrico, pepsina, ácidos biliares e enzimas pancreáticas, o que torna irritante para tecidos não adaptados à sua presença $\mathrm{a}^{(2)}$.

A exposição constante do suco gástrico causa inflamação, edema e ulceração em todo o epitélio respiratório. A passagem do suco gástrico e da pepsina acarreta em prejuízo no funcionamento da tuba auditiva após a exposição constante a $\mathrm{pH}<4$, o qual favorece o aparecimento de otite média ${ }^{(5)}$. Esse tipo de alteração pode ser comum em latentes que apresentam RGE e se submetem a triagem auditiva neonatal (TAN), por meio das emissões otoacústicas. Este exame é sensível à alteração de orelha média, sendo imprescindível a avaliação da mesma (por meio da timpanometria) em neonatos, devido à alta incidência de RGE na faixa etária de zero a 6 meses $^{(6)}$.

A timpanometria é o método mais utilizado na prática clínica para a avaliação das condições funcionais da orelha média, da mobilidade da membrana timpânica e da dinâmica ossicular ${ }^{(7)}$. Os achados timpanométricos são descritos qualitativamente e quantitativamente. Diferentes critérios de interpretação dos resultados podem levar a diferentes conclusões e, consequentemente, a diferentes condutas no diagnóstico, ou seja, detalhes podem ser perdidos se considerarmos os timpanogramas apenas pela sua classificação qualitativa ${ }^{(8)}$. 
Padrões timpanométricos observados em neonatos não estão em conformidade com os padrões clássicos encontrados em crianças e adultos, tornando difícil a aplicação do esquema tradicional de classificação de timpanogramas nos tipos A, B, C, D, As e $\mathrm{Ad}^{(9)}$. Desta forma, para evitar erros ocasionais e interpretações inadequadas, necessita-se de uma complementação com os critérios quantitativos, os quais são influenciados pelos fatores de transmissão presentes no sistema auditivo ${ }^{(8)}$.

A quantificação das medidas timpanométricas facilita o desenvolvimento das normas apropriadas para comparação dos dados timpanométricos entre clínicas e, eventualmente, conduzem os dados de sensibilidade e especificidade que ainda não estão formalizados. As medidas quantitativas são usadas em complemento às medidas qualitativas para caracterizar com maior precisão os timpanogramas. Essas características incluem: Admitância Acústica Estática de Pico Compensado (Ymt), Volume Equivalente do Meato acústico externo da Orelha (Vea), Gradiente do Timpanograma (GR) e Pressão do Pico do Timpanograma (PPT) ${ }^{(8,10)}$. No presente estudo serão abordadas as medidas de compliância e gradiente.

A compliância mede a mobilidade da orelha média, na qual, fatores como massa, rigidez e resistência atuam, facilitando ou impedindo o seu movimento, sendo a mesma, medida em termos de volume equivalente em mililitros ( $\mathrm{ml})$, fornecendo informações que vêm auxiliar outras medidas. Quanto maior a mobilidade, maior a altura do pico; quanto menor a mobilidade, menor a altura do pico. A compliância estática pode ser obtida por meio do cálculo da diferença entre outras duas medidas: o volume da orelha externa e o volume total (volume da orelha externa mais o volume da orelha média).É utilizada para identificar alterações da orelha média, por meio da altura do pico ou da curva timpanométrica que pode aumentar ou diminuir. A altura diminui quando a compliância estática é anormalmente baixa, o que ocorre quando existe rigidez na orelha média e está associada a transtornos como: fixação da cadeia ossicular, colesteatoma, otosclerose e líquido na orelha média, sendo esta última a causa mais comum em crianças. Por outro lado, a compliância estática aumenta quando a admitância é anormalmente alta, que pode resultar de alterações que adicionem massa ao sistema da orelha média ${ }^{(11)}$.

Já o gradiente timpanométrico é uma medida objetiva que descreve a inclinação da forma timpanométrica próxima ao pico. O timpanograma plano pode ser quantificado por meio do gradiente, o qual descreve a relação da sua altura com a sua largura. O gradiente de timpanogramas absolutos pode ser medido em termos de mmhos ou $\mathrm{ml}$, sendo necessário traçar uma linha horizontal no ponto em que a largura do timpanograma é de \pm 100 daPa, daí mede-se a altura do pico sobre essa linha e a altura total do pico até a base, encontrando o gradiente pela divisão da altura do pico e altura total ${ }^{(7)}$. Os valores variam de 0,0 a 1,0 ml. Gradientes menores que 0,2 são considerados anormalmente baixos e estão associados à presença de fluidos na orelha média ${ }^{(12)}$.

Quando se detecta alterações na compliância estática e no gradiente timpanométrico de forma isolada, faz-se necessária uma segunda testagem, porém, quando esta alteração ocorre em ambas as medidas timpanométricas, sugere alterações de orelha média, sendo necessário encaminhamento para exames complementares $^{(13)}$.

A timpanometria convencional é realizada com a sonda de $226 \mathrm{~Hz}$ e os resultados com esta sonda têm considerável valor diagnóstico para idosos, adultos e crianças a partir de 6 meses de idade, porém, em relação a neonatos e lactentes abaixo de 6 meses há controvérsias devido à menor frequência de ressonância e à baixa sensibilidade, ou seja, ao alto índice de falso negativo nessa população ${ }^{(6,7)}$. Neonatos de até 6 meses de idade com efusão confirmada cirurgicamente, quando testados com sondas de baixas frequências, podem apresentar timpanogramas normais de pico único ou de duplo pico ${ }^{(14)}$.

A literatura mostra que a timpanometria com tom de frequência alta $(1 \mathrm{kHz})$ é mais sensível para as alterações de orelha média nos lactentes abaixo de 6 meses do que a timpanometria com tom de sonda de $226 \mathrm{~Hz}^{(6)}$, sendo útil na distinção das falhas ocasionadas por perdas sensorioneurais e patologias condutivas. $\mathrm{O}$ uso da timpanometria com tom de 1 $\mathrm{kHz}$ deve assegurar uma base para a avaliação dos benefícios das diversas opções de tratamento nessa população(7).

Partindo das considerações supracitadas, pode-se ressaltar que é de extrema importância que a curva timpanométrica seja obtida com precisão. A falta de guia para o tratamento das alterações da orelha média é particularmente atribuída, à falta de um teste de diagnóstico efetivo para neonatos menores de 6 meses. Sendo assim, são importantes estudos que comparem as análises das medidas timpanométricas em diferentes sondas, devido à alta incidência de alterações de orelha média em lactentes com refluxo, uma vez que mesmo em curvas timpanométricas normais pode ser observado o alargamento e a diminuição da amplitude da compliância.

Assim, o objetivo do presente estudo foi analisar e correlacionar a compliância nas sondas de $226 \mathrm{~Hz}$ e $1 \mathrm{kHz}$ entre grupos de lactentes com e sem refluxo gastroesofágico, como também analisar e comparar o gradiente timpanométrico na sonda de $226 \mathrm{~Hz}$ entre os grupos.

\section{MÉTODOS}

A coleta de dados iniciou-se após aprovação do Comitê de Ética em Pesquisa da Universidade Estadual de Ciências da Saúde de Alagoas (UNCISAL), sob protocolo número 829. O estudo foi realizado no serviço de Otorrinolaringologia do Instituto da Mulher do Hospital Santa Juliana de Alagoas.

A amostra foi constituída por 118 lactentes com faixa etária de RN até 6 meses, sendo divididos em dois grupos: grupo estudo (GE) e grupo controle (GC). O GE foi composto por 63 lactentes com refluxo gastroesofágico fisiológico, encaminhados por médicos pediatras ou gastropediatras com diagnóstico clínico. O GC foi composto por 55 lactentes sem RGE. O diagnóstico clínico foi realizado por meio de um formulário baseado no protocolo de ROMA II ${ }^{(15)}$, que classifica o RGE quando o lactente apresenta os seguintes sintomas: regurgitação, náuseas, vômitos, retardo ponderal, irritabilidade, choro excessivo, arqueamento, soluços, sintomas respiratórios (tosse, sibilos, estridor, apneia) e distonias. No presente estudo, o RGE foi classificado como fisiológico quando o lactente apresentava pelo menos dois desses sintomas. 
Foram excluídos da pesquisa lactentes com fissura labiopalatina, má formação de orelha externa e/ou média, má formação de cabeça e pescoço e síndromes genéticas associadas a alterações auditivas.

Todos os lactentes desta pesquisa foram submetidos à avaliação otorrinolaringológica antes da realização dos exames auditivos, com Otoscópio Hinne Walchilling $®$ para avaliação das orelhas externa e média.

A timpanometria foi realizada com o analisador de orelha média: Impedance Audiometer - AT235h - Interacoustics®, nas sondas $226 \mathrm{~Hz}$ e $1 \mathrm{kHz}$. Foram analisadas duas medidas timpanométricas: a compliância e o gradiente, sendo a compliância avaliada nos dois tipos de sonda e o gradiente avaliado apenas com a sonda de $226 \mathrm{~Hz}$, ambos obtidos em $\mathrm{ml}$.

O cálculo do tamanho da amostra foi realizado para estudo de corte transversal, comparando o grupo com refluxo e o grupo sem refluxo, atribuindo um erro alfa e beta de 5\%. Para comparação da compliância entre os grupos em cada orelha, nas sondas de $226 \mathrm{~Hz}$ e $1 \mathrm{kHz}$, foi utilizado o teste de Mann-Whitney e para comparação da compliância entre as sondas foi utilizado o teste de Wilcoxon.

\section{RESULTADOS}

Na distribuição dos bebês com relação à variável gênero, observou-se discreto predomínio do gênero feminino, tanto no grupo estudo, como no grupo controle (Tabela 1). Embora tenha existido diferença entre os percentuais dos dois grupos com e sem RGE, as diferenças não foram consideradas significativas.

Com a aplicação do teste de Mann-Whitney foi possível observar diferença na comparação dos resultados da média de

Tabela 1. Comparação dos sujeitos segundo o gênero, nos grupos estudados

\begin{tabular}{|c|c|c|c|c|c|}
\hline \multirow{2}{*}{ Gênero } & \multicolumn{2}{|c|}{ GE } & \multicolumn{2}{|c|}{ GC } & \multirow{2}{*}{$\begin{array}{l}\text { Valor } \\
\text { de } p\end{array}$} \\
\hline & $\mathrm{n}$ & $\%$ & $\mathrm{n}$ & $\%$ & \\
\hline Feminino & 36 & 57,10 & 30 & 54,50 & 0,777 \\
\hline Masculino & 27 & 42,90 & 25 & 45,50 & \\
\hline Total & 63 & 100,00 & 55 & 100,00 & \\
\hline
\end{tabular}

Legenda: $\mathrm{GE}=$ grupo estudo; $\mathrm{GC}=$ grupo controle compliância entre os grupos, tanto na sonda de $226 \mathrm{~Hz}$ como na sonda de $1 \mathrm{kHz}$. Quando utilizada a sonda de $226 \mathrm{~Hz}$, os valores médios de compliância encontrados foram de $0,55 \mathrm{ml}$ em ambas as orelhas no grupo com refluxo. Já no grupo sem RGE os valores foram de: $0,66 \mathrm{ml}$ na orelha direita (OD) e $0,62 \mathrm{ml}$ na orelha esquerda (OE). Na utilização da sonda de 1 $\mathrm{kHz}$, a média de compliância foi de $1,01 \mathrm{ml}$ na OD e $0,86 \mathrm{ml}$ na OE, do grupo com RGE. No grupo sem refluxo observou-se $1,13 \mathrm{ml}$ na OD e $1,06 \mathrm{ml}$ na OE. Ao comparar os resultados das médias da compliância entre os grupos, foi possível observar maiores valores no grupo sem refluxo em ambas as orelhas, independente do tipo de sonda utilizada.

Ao comparar-se os resultados da compliância estática entre as sondas na mesma orelha em cada grupo, observou-se maior compliância com o uso da sonda de $1000 \mathrm{~Hz}$, em todos os lactentes estudados, em relação à sonda de $226 \mathrm{~Hz}$, com diferença significativa (Tabela 2).

Na comparação dos resultados entre os grupos do gradiente timpanométrico foi possível observar diferença entre os grupos para o valor do gradiente, em ambas as orelhas. Foi possível ainda observar que os lactentes com RGE apresentaram médias dos valores do gradiente menores quando comparados ao grupo sem refluxo. No entanto, observou-se também que no grupo sem refluxo, houve valores alterados em Q1 na OD $(0,15)$ e OE $(0,19)$, porém, bem diferentes dos valores do grupo com refluxo (Tabela 3 ).

\section{DISCUSSÃO}

No presente estudo foi encontrado discreto predomínio do RGE no gênero feminino. Em outros estudos, esse discreto predomínio foi encontrado no gênero masculino. Isso pode ter ocorrido devido a amostra dessa população ter sido maior no presente estudo ${ }^{(16-18)}$ (Tabela 1).

No presente estudo os lactentes com RGE obtiveram um padrão de compliância dentro da normalidade, porém, menores quando comparados aos lactentes sem RGE. É possível que o GE apresente uma discreta alteração de orelha média, ainda não detectada pela timpanometria. De acordo com alguns autores $^{(1,2)}$, lactentes com RGE podem apresentar alterações de orelha média (discretas a severas), considerando o valor reduzido da altura timpanométrica, que pode estar associada

Tabela 2. Comparação dos resultados da compliância estática obtidos com a sonda de $226 \mathrm{~Hz}$ e $1 \mathrm{kHz}$ para cada orelha e entre os grupos estudados

\begin{tabular}{|c|c|c|c|c|c|c|c|c|}
\hline \multirow{3}{*}{$\begin{array}{l}\text { Compl. - } \\
\text { Timp. }\end{array}$} & \multicolumn{4}{|c|}{$\mathrm{GE}$} & \multicolumn{4}{|c|}{$\mathrm{GC}$} \\
\hline & \multicolumn{2}{|c|}{ OD } & \multicolumn{2}{|c|}{ OE } & \multicolumn{2}{|c|}{ OD } & \multicolumn{2}{|c|}{$\mathrm{OE}$} \\
\hline & $226 \mathrm{~Hz}$ & $1 \mathrm{kHz}$ & $226 \mathrm{~Hz}$ & $1 \mathrm{kHz}$ & $226 \mathrm{~Hz}$ & $1 \mathrm{kHz}$ & $226 \mathrm{~Hz}$ & $1 \mathrm{kHz}$ \\
\hline Média & 0,55 & 1,01 & 0,55 & 0,87 & 0,66 & 1,13 & 0,62 & 1,06 \\
\hline Mediana & 0,51 & 0,85 & 0,51 & 0,77 & 0,59 & 1,04 & 0,54 & 0,80 \\
\hline DP & 0,32 & 0,90 & 0,35 & 0,74 & 0,31 & 0,66 & 0,29 & 0,77 \\
\hline Q1 & 0,34 & 0,19 & 0,29 & 0,17 & 0,43 & 0,57 & 0,40 & 0,49 \\
\hline Q3 & 0,70 & 1,67 & 0,77 & 1,45 & 0,85 & 1,55 & 0,77 & 1,50 \\
\hline$n$ & 63 & 63 & 63 & 63 & 55 & 55 & 55 & 55 \\
\hline IC & 0,08 & 0,22 & 0,09 & 0,18 & 0,08 & 0,17 & 0,08 & 0,20 \\
\hline Valor de $p$ & \multicolumn{2}{|c|}{$<0,001^{*}$} & \multicolumn{2}{|c|}{$0,003^{*}$} & \multicolumn{2}{|c|}{$<0,001^{*}$} & \multicolumn{2}{|c|}{$<0,001^{*}$} \\
\hline
\end{tabular}

*Valores significativos $(\mathrm{p} \leq 0,05)-$ Teste de Wilcoxon

Legenda: GE = grupo estudo; GC = grupo controle; Compl. = compliância; Timp. = timpanometria; OD = orelha direita; OE = orelha esquerda; DP = desvio-padrão; IC = intervalo de confiança 
Tabela 3. Comparação dos resultados do gradiente timpanométrico obtidos com a sonda de $226 \mathrm{~Hz}$ para cada orelha e entre os grupos estudados

\begin{tabular}{llcccccccc}
\hline Timp (226 Hz) & Média & Mediana & DP & Q1 & Q3 & $n$ & IC & Valor de $p$ \\
\hline \multirow{2}{*}{ Grad. OD } & Com RGE & 0,20 & 0,12 & 0,27 & 0,07 & 0,24 & 63 & 0,07 & $<0,001^{*}$ \\
& Sem RGE & 0,28 & 0,23 & 0,26 & 0,15 & 0,30 & 55 & 0,07 & 0,03 \\
\multirow{2}{*}{ Grad. OE } & Com RGE & 0,18 & 0,14 & 0,14 & 0,09 & 0,25 & 63 & $0,001^{*}$ \\
& Sem RGE & 0,25 & 0,23 & 0,11 & 0,19 & 0,30 & 55 & 0,03 & \\
\hline
\end{tabular}

*Valores significativos $(p \leq 0,05)$ - Teste de Mann-Whitney

Legenda: Com RGE = com refluxo; Sem RGE = sem refluxo; Grad. = gradiente; $\mathrm{OD}$ = orelha direita; $\mathrm{OE}=$ orelha esquerda; desvio-padrão; IC = intervalo de confiança

à menor mobilidade do sistema tímpano-ossicular( ${ }^{(7)}$.

Quando realizada a comparação entre as sondas de 226

$\mathrm{Hz}$ e $1 \mathrm{kHz}$ (Tabela 2), a sonda de $1 \mathrm{kHz}$ apresentou valores médios de compliância maiores em relação à sonda de 226 $\mathrm{Hz}$, corroborando estudo ${ }^{(19)}$ realizado em 110 bebês de 6 a 30 dias de idade. Isso pode ser explicado pela frequência de ressonância na orelha média do neonato, bem como pelo sistema dominado por massa nesta faixa etária ${ }^{(7)}$.

Esses achados estão de acordo ainda com o estudo que analisou e comparou médias timpanométricas nas sondas de $226 \mathrm{~Hz}$ e $1 \mathrm{kHz}$, encontrando valores médios de compliância estática de $0,85 \mathrm{ml}$ na sonda de $1 \mathrm{kHz}$ e $0,37 \mathrm{ml}$ na sonda de $226 \mathrm{~Hz}$, observando diferença significativa ${ }^{(20)}$. Em outro estudo, foram analisados os efeitos da timpanometria de $226 \mathrm{~Hz}, 678$ $\mathrm{Hz}$ e $1 \mathrm{kHz}$ em um grupo de crianças com Potencial Evocado Auditivo de Tronco Encefálico (PEATE) normal e outro com PEATE sugestivo de perda auditiva condutiva na faixa de cinco a 25 semanas. Para a frequência de $226 \mathrm{~Hz}$ não se observou nenhuma diferença entre a compliância estática em ambos os grupos avaliados. Os timpanogramas realizados com sonda de $678 \mathrm{~Hz}$ demonstraram variados picos e diversos tipos de curvas em ambos os grupos, enquanto que com a sonda de 1 $\mathrm{kHz}$ foram encontrados no grupo normal, timpanogramas com único pico e, no grupo alterado, timpanogramas sem pico. Os autores concluíram que o uso da timpanometria com sonda de $1 \mathrm{kHz}$ apresentou resultados mais precisos na avaliação da função de orelha média de crianças com menos de 25 semanas $^{(21)}$.

Devido ao alto índice de resultados rebaixados do gradiente timpanométrico no grupo com refluxo, foi observado que quando realizada a média desses resultados, o grupo estudo apresentou valores limítrofes e alterados (Tabela 3), indicando a presença de comprometimento no que diz respeito ao sistema tímpano-ossicular, como efusão de orelha média. Foram obser- vados ainda, maiores valores de gradiente na OD de ambos os grupos, corroborando alguns autores ${ }^{(7)}$ que encontraram valores médios de compliância estática e de gradiente timpanométrico significantemente mais altos na orelha direita em relação à esquerda. Um dado que chamou a atenção, foi o valor alterado de Q1 no grupo sem refluxo, e isso pode ter ocorrido devido ao desvio-padrão da medida ter sido elevado. Em sujeitos com alterações de orelha média, o gradiente encontra-se menor que 0,2 e o timpanograma mais achatado, sendo classificado como curva com "pico arredondado". Sua análise, muitas vezes, é difícil, principalmente nos casos limítrofes, ou seja, nas curvas que apresentam compliância rebaixada com valores de gradiente limítrofes ${ }^{(22,23)}$.

Diante da dificuldade de interpretação de alguns timpanogramas com compliância rebaixadas ou gradientes alterados ou limítrofes, faz-se necessário o encaminhamento dessa população para exames complementares, como emissões otoacústicas e potencial evocado auditivo de tronco encefálico por via aérea e via óssea, bem como uma avaliação otorrinolariongológica específica, no intuito de confirmar possíveis alterações de orelha média e de iniciar tratamento imediato.

\section{CONCLUSÃO}

A compliância é maior na sonda de $1 \mathrm{kHz}$, tanto em lactentes com RGE como sem RGE, quando comparada com a sonda de $226 \mathrm{~Hz}$. Lactentes com refluxo apresentam compliância dentro dos padrões de normalidade para integridade de orelha média, porém, apresentam menores compliâncias quando comparados com lactentes sem refluxo. Com relação ao gradiente, lactentes com RGE apresentam valores do gradiente alterados e/ou dentro dos padrões limítrofes da normalidade em ambas as orelhas.

\begin{abstract}
Purpose: To analyze and compare the tympanometric gradient and the compliance obtained in probe tones of 226 and $1 \mathrm{kHz}$ between groups of infants with and without gastroesophageal reflux (GER). Methods: Participants were 118 full-term and preterm infants, from newborns to 6-month-olds - 63 with clinical diagnosis of physiological GER performed by pediatricians or gastroenterologists, and 55 without GER -, who were submitted to tympanometry with probe tones of 226 and $1 \mathrm{kHz}$. Results: Higher compliance values were found with $1 \mathrm{kHz}$ in both groups. Comparing the average compliance between groups, we observed that the group without reflux showed higher values. The mean value of the tympanometric gradient was higher in the group without reflux, when compared to the one with reflux. Conclusion: The $1 \mathrm{kHz}$ probe tone has higher compliance in infants with and without GER, when compared to the $226 \mathrm{~Hz}$ probe tone. Infants with reflux have compliance within normal limits, although they present lower compliance than infants without reflux. Regarding the gradient, infants with GER have altered values and/or within normality limits in both ears.
\end{abstract}

Keywords: Acoustic impedance tests; Ear, middle; Gastroesophageal reflux; Infant; Hearing 


\section{REFERÊNCIAS}

1. Gaynor EB. Otorrinolaryngologic manifestations of gastroesophageal Reflux. Am J Gastroenterol. 1991;86(7):801-8.

2. Nelson SP, Chen EH, Syniar GM, Christoffel KK. Prevalence of symptoms of gastroesophageal reflux during childhood: a pediatric practice-based survey. Pediatric Practice Research Group. Arch Pediatr Adolesc Med. 2000;154(2):150-4.

3. Hillemeier AC. Gastroesophageal reflux. Diagnostic and therapeutic approaches. Pediatr Clin Nort Am. 1996;43(1):197-212.

4. Norton RC, Penna FJ. Refluxo gastroesofágico. J Pediatr (Rio J). 2000;76(Supl 2):S218-24.

5. Crapko M, Kerschner JE, Syring M, Johnston N. Role of extraesophageal reflux in chronic otitis media with effusion. Laryngoscope. 2007;117(8):1419-23.

6. Garcia MV, de Azevedo MF, Testa JR. Medidas de imitância acústica em lactentes com 226hz e 1000hz: correlação com as emissões otoacústicas e o exame otoscópico. Rev Bras Otorrinolaringol. 2009;75(1):80-9.

7. Lyra e Silva KA, Novaes BA, Lewis DR, Carvallo RM. Achados timpanométricos em neonatos com emissões otoacústicas presentes: medidas e interpretações. Rev Bras Otorrinolaringol. 2007;73(5):633-9.

8. Northern J, Downs M. Hearing in children. 5th ed. Philadelphia: Lippincott, Williams \& Wilkins; 2002. Acoustic immittance assessment; p. 217-52.

9. Jerger J. Clinical experience with impedance audiometry. Arch Otolaryngol. 1970;92(4):311-24.

10. Fowler CG, Shanks JE. Tympanometry. In: Katz J, editor. Handbook of clinical audiology. 5th ed. Philadelphia: Lippincott Williams \& Wilkins; 2002. p.175-204.

11. Santos TM, Russo IC.. A prática da audiologia clínica. São Paulo: Cortez.; 1991. Logoaudiometria; p. 73-88.

12. Carvallo RM. Medidas de imitância acústica em crianças. In: Lichtig I, Carvallo RM. Audição abordagens atuais. Pró-Fono. Carapicuíba: PróFono; 1997. p.67-87.
13. Reis AC, Zorzi GB. Valores de gradiente timpanométrico em crianças com faixa etária de 4 a 10 anos. Rev Cient Univ Franca. 2005;5:223-8.

14. Margolis RH, Bass-Ringdahl S, Hanks WD, Holte L, Zapala DA. Tympanometry in newborn infants: $1 \mathrm{kHz}$ norms. J Am Acad Audiol. 2003;14(7):383-92.

15. Roma Foundation.org. Roma Foundation introduces Diagnostic Algorithms for Common Gastrointestinal Symptoms, Inc [Internet]. McLean, VA: c2009 [updated 2009 Fev 10; cited 2009 Mar 9]. Rome III Diagnostic Criteria for Functional Gastrointestinal Disorders. Available from: http://www.romecriteria.org/education/clin_algor_project.cfm

16. Costa AJ, Silva GA, Gouveia PA, Pereira Filho EM. Prevalência do refluxo gastroesofágico patológico em lactentes regurgitadores. J Pediatr (Rio J). 2004;80(4):291-5.

17. Oliveira NF, Norton RC. Relação entre achados videonasolaringoscópicos e pH-metria esofágica em crianças com manifestações clínicas de refluxo extra-esofágico. Arq Int Otorrinolaringol. 2009;13(2):140-5.

18. Teele DW, Klein JO, Rosner BA. Epidemiology of otitis media in children. Ann Otol Rhinol Laryngol Suppl. 1980;89(3Pt 2):5-6.

19. Linares AE, Carvallo RM. Medidas imitanciométricas em crianças com ausência de emissões otoacústicas. Rev Bras Otorrinolaringol. 2008;74(3):410-6.

20. Stumpf CC. Medidas de imitância acústica em neonatos [dissertação]. Curitiba: Universidade Tuiuti do Paraná; 2008.

21. Shang YY, Ni DF, Liu SL. [Comparison of the low and high frequency tympanometries as diagnostic tests of middle ear function in infants]. Zhonghua Er Bi Yan Hou Tou Jing Ke Za Zhi. 2006;41(5): 326-30.

22. Almeida RN. Processos de medidas da imitância acústica [monografia]. Rio de Janeiro: Centro de especialização em Fonoaudiologia Clínica; 1999.

23. Carvallo RM. Medidas de imitância acústica em crianças. In: Lichtig I, Carvallo RM. Audição: abordagens atuais. São Paulo: Pró-Fono; 1997. p.90-117. 